\title{
What is good drinking water? 41 years of water conflicts around the Nuclear Research Centre Karlsruhe, 1956-1997
}

\author{
Alicia Gutting \\ Division of History of Science, Technology and Environment, KTH Royal Institute of Technology, \\ Stockholm, 100 44, Sweden \\ Correspondence: Alicia Gutting (gutting@kth.se) \\ Published: 10 November 2021
}

\begin{abstract}
Drinking water in Germany is usually praised as the best-controlled food that can be enjoyed almost without exception. What constitutes high-quality drinking water is defined by law and drinking water should "inspire enjoyment, in other words, it should be colourless, clear, cool and odourless as well as tasty" (Deutsches Institut für Normung, 2017, DIN 2000:2017-02). Since this food is one of the basic human needs, it receives special social attention. When in 1956, the idyllic Rhine valley in the borderlands between the south west of Germany and France was turned into the nuclear capital of Germany with the siting of the Nuclear Research Centre in Leopoldshafen north of Karlsruhe, the local community feared especially a reduction of the water quality. This early perception of water risks ran like a thread through the history of the Nuclear Research Centre and the local population. This paper traces back the long-lasting conflict between the people in the Hardtwald area, where Karlsruhe and Eggenstein-Leopoldshafen are located, who valued their rural surroundings and lived mostly from agriculture in the 1950s and the claims as well as hopes of the Federal Republic of Germany, which saw the federal reactor station as central for the country's future flourishment in the post-war period (Gleitsmann, 2011). This clash of values between the Nuclear Research Centre, the different governments and the people of the Hardtwald area continued up until the 1990s, when the municipality of Eggenstein-Leopoldshafen made plans to take over one of the water wells of the Nuclear Research Centre. The takeover became a strategic component in the municipality's Water Concept 2000, through which it aimed at modernising and securing its drinking water supply for the future. During the hearing, opponents cited past violations of rules and free interpretations of threshold values as the basis for their counter arguments. This body of knowledge from the past was knowledge lived by the opponents of the Water Concept 2000. The background information from 41 years shaped their risk perception.

Historicizing risk is valuable in the way of gaining a deeper understanding of local resistance against nuclear sites. Risk perception is not a linear process and relies heavily on communication processes as well as the recognition of different value systems. Social science research on resistance against nuclear siting often remains in the moment, even though historians have shown that there is a deeper history behind the opposition. Additionally, historical research often lacks both a theorisation and a conceptualisation of the issues portrayed. Seen from a wider perspective, sociologically informed historical research can contribute to future decision-making concerning nuclear sites, such as nuclear waste storage as well as other technological sites perceived as being risky. Being able to understand where increased risk perceptions come from, how increased resistance occurred and also which mistakes could have been avoided, paves the way for understanding cooperation and for finding sustainable solutions.
\end{abstract}

Kurzfassung. Trinkwasser wird in Deutschland gewöhnlich als das am besten kontrollierte Lebensmittel gepriesen, das beinahe ausnahmslos genossen werden kann. Was Trinkwasser von hoher Qualität ausmacht, ist im Gesetz definiert, Trinkwasser sollte ,appetitlich sein und zum Genuss anregen. Es muss farblos, klar, kühl 
sowie geruchlich und geschmacklich einwandfrei sein“ (Deutsches Institut für Normung, 2017, DIN 2000:201702). Da dieses Lebensmittel eines der grundlegenden menschlichen Bedürfnisse darstellt, erhält es besondere soziale Aufmerksamkeit. Als im Jahr 1956 das idyllische Rheintal im Grenzgebiet zwischen dem Südwesten Deutschlands und Frankreich mit der Ansiedlung des Kernforschungszentrums in Leopoldshafen nördlich von Karlsruhe in die nukleare Hauptstadt von Deutschland verwandelt wurde, befürchtete die Gemeinde vor Ort insbesondere eine Abnahme der Wasserqualität. Diese frühe Wahrnehmung von Risiken für das Wasser spann sich wie ein roter Faden durch die Geschichte des Kernforschungszentrums und der örtlichen Bevölkerung. In der vorliegenden Arbeit rückverfolgt die Autorin die Spuren des langanhaltenden Konflikts zwischen den Menschen im Hardtwaldgebiet, in dem Karlsruhe und Eggenstein-Leopoldshafen liegen, die ihre ländliche Umgebung wertschätzten und in den 1950er-Jahren zumeist von der Landwirtschaft lebten, und den Ansprüchen und Hoffnungen der Bundesrepublik Deutschland, die die deutsche Reaktorstation als zentral für das zukünftige Gedeihen des Landes in der Nachkriegszeit ansah (Gleitsmann, 2011). Dieser Wertekonflikt zwischen dem Kernforschungszentrum, den unterschiedlichen Regierungen und den Menschen aus dem Hardtwald setzte sich bis in die 1990er-Jahre fort, als die Gemeinde von Eggenstein-Leopoldshafen Pläne erstellte, um einen der Wasserbrunnen des Kernforschungszentrums zu übernehmen. Die Übernahme wurde zu einem strategischen Bestandteil im Wasserkonzept 2000 der Gemeinde, mit dem sie darauf abzielte, ihre Trinkwasserversorgung für die Zukunft zu modernisieren und zu sichern. Während der Anhörung nannten Gegner und Gegnerinnen frühere Verstöße gegen Vorschriften und freie Interpretationen von Grenzwerten als die Grundlage für ihre Gegenargumente. Dieser Wissensbestand aus der Vergangenheit war gelebtes Wissen der Gegner und Gegnerinnen des Wasserkonzepts 2000. Die Hintergrundinformationen aus 41 Jahren hatten ihre Risikowahrnehmung geprägt.

Die Historisierung von Risiken ist wertvoll im Sinne der Erlangung eines tieferen Verständnisses des lokalen Widerstands gegen nukleare Standorte. Risikowahrnehmung ist kein linearer Prozess und stützt sich stark auf Kommunikationsprozesse sowie die Anerkennung verschiedener Wertesysteme. Häufig verbleibt die sozialwissenschaftliche Forschung zum Widerstand gegen die Standortwahl für nukleare Anlagen im Augenblick, obwohl Historiker und Historikerinnen nachgewiesen haben, dass eine tiefere Geschichte hinter dem Widerstand steckt. Darüber hinaus fehlt es der historischen Forschung oftmals sowohl an Theoriebildung als auch an Konzeptualisierung der dargestellten Fragestellungen. Aus größerer Perspektive betrachtet kann die soziologisch informierte historische Forschung zur künftigen Entscheidungsfindung hinsichtlich nuklearer Standorte, wie Lagerstätten für nukleare Abfälle, sowie anderer als risikoreich wahrgenommener Technologiestandorte beitragen. Wenn man in der Lage ist zu verstehen, woher erhöhte Risikowahrnehmungen stammen, wie es zu einem vermehrten Widerstand kam, aber auch, welche Fehler hätten vermieden werden können, ebnet das den Weg für eine verständnisvolle Kooperation und für die Suche nach nachhaltigen Lösungen.

Financial support. This research has been supported by the European Research Council, H2020 European Research Council (grant no. NUCLEARWATERS (771928)).

\section{References}

Deutsches Institut für Normung: Zentrale Trinkwasserversorgung Leitsätze für Anforderungen an Trinkwasser, Planung, Bau, Betrieb und Instandhaltung der Versorgungsanlagen, Beuth, Berlin, 2017.

Gleitsmann, R. J.: Der Vision atomtechnischer Verheißungen gefolgt: Von der Euphorie zu ersten Protesten - Die zivile Nutzung der Kernkraft in Deutschland seit den 1950er Jahren, J. New Front. Spat. Concept., 3, 17-26, 2011. 